present will separate out, and must be removed by filtration. The filtrate is now evaporated to $50 \mathrm{c.c}$., and this washed out of the basin into a special form of measuring tube, and made up to a volume of 60 c.c.; 25 c.c. of concentrated hydrochloric acid are now added, with constant stirring, followed by a few c.c. of sodium chloride. The phenols will now be disengaged and rise to the surface, and their volume read off at $60^{\circ} \mathrm{F}$ 'ahr, this giving the amount of commercial carbolic acid in 75 grams. The process has to be somewhat modified when the powder to be examined has a lime base, as the phenols are then combined. In that case, before treating with alcohol, small quantities of dilute sulphuric acid are added, until the powder has a distinct though taint acid reaction (care being taken that none of the powder escapes the action of the acid), when the process is applied as above described.

Soxhlet was the first to suggest that the addition of minute quantities of phenolphthalein to margarine and mixtures of butter and margarine should be made compulsory, in order to afford a ready means of proving whether a sample of butter was genuine or not, for on touching a sample so treated with an alkali the characteristic red-violet colour is immediately developed. Objection was taken to his proposal on the ground that the keeping qualities of the margarine were, in consequence, considerably impaired. Hart has recently carried out some investigations to elucidate this, and has shown that when phenolphthalein is added to margarine in proportions of 2 to 6 milligrammes to $5 \circ$ grams of margarine, no deterioration took place after six weeks' exposure to light and air. With a view of finding out whether it had any toxic effect he took doses of from I to 20 milligrammes without ill effect, but on increasing the dose to $0^{\circ} 3$ gramme he suffered from diarrhoea, which became more severe when the quantity waa increased to 0.5 gramme. When taken in such quantities as these the margarine had a distinct bitter taste. He strongly approved of Soxhlet's proposal, as no ill effects were produced by such small quantities as were needful for this purpose.

SMald-POX IN FRANCE-A short statement bas been made by M. Hervieux in the "Acad. de Méd." on the epidemic of small-pox during 1892 and r893. It appeared chiefly in and around Paris, but with subsidiary outbreaks in the provinces. During the first six months of 1892 I4 deaths were reported in Paris ; in the last six months 26 deaths. In the first six months of $1893 \quad 63$ deaths were reported, and in the second six months 184 . The outbreak is attributed to the absence of revaccination, and the presence of small-pox in England; this being supported by the heavy mortality in the Pas de Calais and neighbouring departments.-Sem. $M \ell d$.

\section{ON AN OUTBREAK OF SMALL-POX IN} AN ISOLATION HOSPITAL,

\section{Apparently CaUSed by INfected Steam from A LAUNDRY.*}

By Geo. WILson, M.A., M.D., M.O.H. for the Mid. Warwickshire Combined Sanitary District.

IT has been my duty, in conjunction with Dr. Bernays, medical officer, to inquire into four cases of small-pox, all of which had contracted the disease in the infectious hospital for Solihull while convalescing from scarlatina. The facts are these: Two of the patients, aged respectively I4 years and 25 , were discharged cured, the one on January 23 rd and the other on the $25^{t b}$, and were readmitted suffering from small-pox of a mild type, the one on January 3 oth and the other on February 3 rd, with the rash well-developed. It was ascertained that this second case fell ill on January 3 Ist. The other two cases, aged respectively 24 and I 2 years, sickened with smallpox on January 28 th and 29 th respectively.

In the beginning of January there were no smallpox cases in hospital, but on the Ioth a man aged 20 years was admitted suffering from malignant small-pox, and died on January $r 8$ th. Two other cases were admitted on January $x_{5}$ th ; on the 21 st another severe confluent case, and on January 29th and 3 Ist two more cases were admitted. Thus apart from the cases which contracted the disease in hospital there were six others under treatment in the new block at the close of the month.

It was ascertained that the first patient with a malignant attack had never been vaccinated, and certainly showed no vaccination marks. The smell from this patient was so very intense and offensive that the windows of the ward had always to be kept open.

Now the cases which contracted the disease in hospital sickened on January $28 \mathrm{th}, 29$ th, or 3oth. The washerwoman attached to the hospital also sickened with small-pox on the 29 th. This woman was revaccinated last year, but unsuccessfully.

The whole of these five cases falling ill between January 28 th and 3 oth proves that the infecting agency was common to all, and presumably took effect on the same day and between the $I 7$ th and I8th. As the case which proved fatal on the 18 th was admitted on January roth and buried on the 2oth, any infection which might be conveyed from the dead body is excluded as a common agent on account of the time allowed for incubation being too short. Apart from the possibility of aërial connection - that is, transmission of infection through the air from the new block direct, the frequency of which is strongly maintained by the

\footnotetext{
* From a report to the Solihall Rural Sanitary Authority.
} 
medical department of the Local Government Board-we ascertained that the first washing from the small-pox block, including the bed sheets and linen of the malignant case, was done on the 17 th. Distinct rules were laid down by the medical officer as to the steeping of these articles in a strong disinfectant solution before being taken to the washhouse, and separate days were also assigned to the washing of linen from the scarlatina block and the new block in which the small-pox cases were treated. It was succinctly stated to us that the scarlatina washing was done on January $I 5^{\text {th, the }}$ clothing then washed not having been distributed to the patients for wear till the 2 xst. If this statement is correct it excludes the probability of infection being conveyed directly by mixing articles of clothing or bedding from the two blocks in the washhouse. The fact, however, that the washerwoman contracted the disease on or about the $I 7$ th, when the small-pox linen was first washed, and that this date corresponds approximately to the incubation period of the other cases, affords a strong presumption in our minds that the disinfection of the clothing from the small-pox cases was insufficient, and that when the articles were put into the copper the steam developed before the boiling point was reached, and wafted with it the infective germs of the disease. The washhouse is situated about 30 feet from the scarlatina block in which the patients contracted the disease, while the distance from this block to the new (small-pox) block is about 90 feet. Although, therefore, it is possible that the infection of the disease may have been conveyed direct from one block to the other, we are inclined to accept the view that the infection was conveyed in the vapour given off by the copper in the washbouse, which lies midway between the two blocks, though not in a direct line. It was ascertained, too, that the prevailing winds were from the direction of the new block and washhouse during the whole of the middle and latter end of January. Further, the washerwoman complained at the time of the very offensive smell given off by the sheets and linen received from the small-pox block.

We inquired carefully into all other causes, such as personal intercourse and interchange of closet pails and coal boxes, but though we are not prepared to say that the rules and instructions have been carried out as strictly as could be desired, we do not believe that the cause of the outbreak lies in any of these directions.

It must be remembered that the resources of the hospital have of late been seriously strained, and that the extensions and alterations have from time to time been interrupted and are not yet completed. We believe that if the disinfecting apparatus had been ready for use this outbreak would not in all probability have occurred. The hospital has done excellent service during the last seventeen years, and though we have repeatedly had sroall-pox under treatment in the small separate block forming part of the old hospital buildings during these years, and three times last year, we have hitherto escaped any such accident as has now occurred.

The nursing staff has all along been adequately provided, and we now say that the nursing in the different blocks is entirely separate, and that there has all along been complete separation of patients suffering from different diseases.

Dr. Wricon, in forwarding the preceding report, remarks that his conclusions as to the probable cause of the outbreak are based not only on the facts as stated, bat he has a vivid recollection of an outbreak of small-pox in a small town a good many years ago, in which cases were traced to the burning of the bedding and clothing of a fatal case in a small garden in a somewhat crowded locality. The fatal case was the first cause of the outbreak. No other cases cropped up until within eleven to fourteen days after the burning of the bedding and clothing, and these were in the near neighbourhood of the infected house. Instructions had been given to pour paraffin over the clothing and bedding before burning, but on subsequent inquiry there were grave doubts whether these had been complied with. Instructions were also given to envelope the dead body in a sheet wrung out of a strong solution of carbolic acid, and also to throw in a large quantity of chloride of lime into the coffin before nailing the lid down. There was great reason to fear that these instructions had also been neglected, because after the incubation interval several cases cropped up in the streets through which the dead body had been conveyed in a hearse, and apart from the fatal case they were all of them first cases. He was so impressed with the lessons of this outbreak that he has never since ordered the bedding or clothing of small-pox cases to be burned, but where there is no hospital accommodation or proper disinfecting apparatus, always to be burned with proper precautions, while the greatest care should be taken in the disinfection of dead bodies by enveloping them completely in a sheet wrung out of perchloride of mercury solution, and if possible enclosing them in an air-tight metallic shell. He further believes that while there can be no doubt of aêrial connection as a cause of the prevalence of small-pox in the neighbourhood of isolation hospitals, he is of opinion that inasmuch as many severe cases of the disease are not removed until they are in a highly infectious stage, the aërial connection is more likely to originate from the ambulance as it passes through crowded thoroughfares than at long distances from the hospital; and the same remarks would apply to the trail of a hearse conveying a dead body which had not been properly disinfected or hermetically sealed up. Then, too, it is well known that the ambulance, if it presents an appearance different from an ordinary vehicle, is always more or less an object of curiosity en route; it may be brought to a stand-still where there is a block in the traffic, and it is always a difficult matter to keep prying neighbours at a safe distance when a patient is about to be removed. In localities where disinfecting stations have been provided there are the further risks attending the removal of highly infected bedding and clothing to be disinfected. He ventures to suggest that all these are factors which contribute far more largely to the theory of aërial connection than the mere aggregation of small-pox cases in an isolation hospital situated in a populous neighbourhood. At the same time he admits that the limited outbreak in the Solihull district fully confirms the policy of the Local Government Board in discountenancing the reception and treatment of small-pox patients on the same site and under the same administrative arrangements as other cases of infectious disease. But in comparatively small districts provided with isolation hospitals, outbreaks of small-pox are so rare that the risks are faced, and, as a rule, without accident. 